\title{
Über dieAutoren
}

Dr. med. Konstanze Loddenkemper ist Ärztin an der Medizinischen Klinik III mit Schwerpunkt Rheumatologie und klinische Immunologie, Universitätsklinikum Charité, HumboldtUniversität zu Berlin. Forschungsschwerpunkte: Steroidinduzierte Osteoporose und Besonderheiten des Knochenstoffwechsels bei Patienten mit entzündlich-rheumatischen Erkrankungen.

Dr. med. Timo Ulrichs ist angehender Facharzt für Mikrobiologieund arbeitet derzeit am Max-Planck-Institut für Infektionsbiologie, Berlin, und am Institut für Infektionsmedizin, Freie Universität Berlin. Forschungsschwerpunkte: Charakterisierung der Immunantwort bei Tuberkulose im Menschen, Impfstoffentwicklung.Außerdem interessiert er sich für Grafik und wissenschaftliche Illustrationen.

Prof. Dr. med. Dr. med. habil. Gerd-Rüdiger Burmester ist Universitätsprofessor für Innere Medizin und Rheumatologie an der Charité, Humboldt-Universität zu Berlin. Für seine bisherige Arbeit erhielt er seit 1977 acht wissenschaftlichePreise. Seit 2001 ist er Präsident der Deutschen Gesellschaft für Rheumatologie. Forschungsschwerpunkte: Klinische und experimentelle Rheumatologie//mmunologie, Immuntherapie, TissueEngineering. 